\title{
Secure Relay and Jammer Selection for Physical Layer Security
}

\author{
Hui Hui, A. Lee Swindlehurst, Fellow, IEEE, Guobing Li, Member, IEEE, and Junli Liang, Member, IEEE
}

\begin{abstract}
Secure relay and jammer selection for physical-layer security is studied in a wireless network with multiple intermediate nodes and eavesdroppers, where each intermediate node either helps to forward messages as a relay, or broadcasts noise as a jammer. We derive a closed-form expression for the secrecy outage probability (SOP), and we develop two relay and jammer selection methods for SOP minimization. In both methods a selection vector and a corresponding threshold are designed and broadcast by the destination to ensure each intermediate node knows its own role while knowledge of the relay and jammer set is kept secret from all eavesdroppers. Simulation results show the SOP of the proposed methods are very close to that obtained by an exhaustive search, and that maintaining the privacy of the selection result greatly improves the SOP performance.
\end{abstract}

Index Terms-Cooperative jamming, physical layer security, relay selection, secrecy outage probability.

\section{INTRODUCTION}

$\mathbf{S}$ ECURITY and privacy are of great importance in modern wireless communications. Traditional cryptographic mechanisms [1] require private key exchange, which introduces additional complexities and redundancy. Physical-layer security, which ensures secure communication by taking advantage of the physical characteristics of wireless channels, has attracted increasing attention. Wyner revealed that when the channel of the legitimate user has better propagation conditions than that of the eavesdropper, secret data transmission is theoretically possible without sharing any key [2]. This conclusion was extended to Gaussian wire-tap channels in [3]. However, when channel conditions in wireless networks are not favorable for the legitimate user, the secrecy rate can be very low or even decline to zero.

Manuscript received November 21, 2014; accepted December 17, 2014. Date of publication January 06, 2015; date of current version January 16, 2015. This work was supported in part by the National Natural Science Foundation of China under Grants 61102061 and 61102079, the National 863 Program of China under Grant 2014AA01A707, and by the Youth Innovation Union of Xi'an University of Technology. The associate editor coordinating the review of this manuscript and approving it for publication was Prof. Chandra Ramabhadra Murthy

H. Hui and J. Liang are with the School of Automation and Information Engineering, Xi'an University of Technology, Xi'an, China. This work was performed during H. Hui's visit to the University of California, Irvine (e-mail: huihui@xaut.edu.cn; liangjunli@xaut.edu.cn).

A. L. Swindlehurst is with the Center for Pervasive Communications and Computing, University of California, Irvine, CA 92697 USA (e-mail: swindle@uci.edu).

$\mathrm{G}$. Li is with the School of Electronics and Information Engineering, Xi'an Jiaotong University, Xi'an, China (e-mail: gbli@mail.xjtu.edu.cn).

Color versions of one or more of the figures in this paper are available online at http://ieeexplore.ieee.org.

Digital Object Identifier 10.1109/LSP.2014.2387860
An efficient solution to meet this challenge is to enhance the legitimate transmission by cooperative relaying or confuse the eavesdroppers via cooperative jamming. In [4], several cooperative relaying schemes are proposed for improving the security of wireless communications, and the security benefits of cooperative jamming have also been extensively studied [5], [6], [7]. The combined use of relays and jamming has also been addressed in a number of papers, including [8]-[17]. More relevant to this paper is prior work that focuses on relay or jammer selection for amplify-and-forward (AF) and decode-and-forward (DF) protocols to improve security in terms of intercept probability, secrecy outage probability or achievable secrecy rate [11], [12], [13]. Selection for the two-way relay case has also been considered in [14] for one eavesdropper whose instantaneous CSI is known. These prior works either ignore the issue of how the selection results are made known to the relays, or they assume that the results are openly broadcast such that the eavesdroppers are aware of them. If the eavesdroppers are aware of the relay and jammer selection, they could cooperate to aggregate the signals of interest and cancel the jamming noise through coordinated beamforming such as interference zero-forcing, and this can dramatically reduce the secrecy of the network. To avoid this impairment, it is critical to keep information about the relay and jammer selection secret from the eavesdroppers.

In this letter, we study secret relay and jammer selection for physical-layer security. We assume a decode-and-forward (DF) relay system, in which a source communicates to a destination with the help of multiple intermediate nodes in the presence of multiple passive eavesdroppers. The intermediate nodes act as either conventional relays or as jammers to hinder the eavesdroppers from intercepting the signal of interest. A closed-form expression for the secrecy outage probability (SOP) is derived for this scenario, and two relay and jammer selection methods are proposed. In these methods, the destination, which is aware of the channel state information (CSI) for the legitimate channels but only the statistics of the eavesdroppers' links, chooses appropriate relay and jammer sets to minimize the SOP. Then, the destination broadcasts information that allows the intermediate nodes to determine whether they will serve as relays or jammers, but this information does not allow the eavesdroppers to know the selection result.

\section{System Model AND TRANSMission Scheme}

\section{A. System Model}

Consider a wireless network consisting of one source $S$, one destination $D$, and $M$ intermediate nodes $T_{1}, T_{2}, \cdots, T_{M}$, in the presence of $K$ passive eavesdroppers $E_{1}, E_{2}, \cdots, E_{K}$. The source, the intermediate nodes and the eavesdroppers are 
equipped with a single antenna, while $D$ has $N$ antennas. We assume that direct links $S$-to- $D$ and $S$-to- $E_{j}$ are not available [12], so data transmission from $S$ to $D$ requires the help of the intermediate nodes. Each intermediate node can act as either a conventional relay to assist in message transmission, or as a jammer that transmits noise to interfere with the eavesdroppers. During each transmission, the intermediate nodes are to be divided into two sets - the relay set $\mathcal{R}$ and the jammer set $\mathcal{J}$.

Let $h_{i, j}$ and $g_{i, j}$ denote the channel coefficient from $T_{i}$ to the $j$ th antenna of the destination, and from $T_{i}$ to $E_{j}$, respectively. All channel coefficients are assumed to be independent and $h_{i, j} \sim \mathcal{C N}\left(0, \lambda_{h_{i, j}}\right), g_{i, j} \sim \mathcal{C N}\left(0, \lambda_{g_{i, j}}\right)$, where $\mathcal{C N}(\mu, \lambda)$ denotes a complex Gaussian distribution with mean $\mu$ and variance $\lambda$. For simplicity we assume that the intermediate nodes are close enough together so that $\lambda_{g_{i, j}}=\lambda_{g_{j}}$. We assume reciprocal channels and that each node has knowledge of the instantaneous CSI of its direct links; i.e., the uplink and downlink channel coefficients from $T_{i}$ to $D$ are identical and available at both $T_{i}$ and $D$. However, only statistical CSI for the eavesdroppers is available at $D$, and no information about the eavesdroppers is available to the intermediate nodes. While the eavesdroppers know $g_{i, j}$, we assume they are unaware of $h_{i, j}$; this is reasonable for time-division duplex scenarios with reciprocal channels, where channel feedback is unnecessary. Finally, we also assume additive white Gaussian noise of power $N_{0}$ is present at $D$ as well.

\section{B. Transmission Scheme}

The transmission is divided into two phases. In phase I, the source transmits a signal $s$. As in [12], all intermediate nodes decode the message successfully in this phase, and the transmission is assumed to be secure in phase I due to the lack of direct links between the source $S$ and the eavesdropper $E_{j}$.

For phase II, define the transmission matrix as $\mathbf{H}=$ $\left[\mathbf{h}_{1}, \mathbf{h}_{2}, \ldots, \mathbf{h}_{M}\right]$, where each column of $\mathbf{H}$ is defined as $\mathbf{h}_{i}:=\left[h_{i, 1}, h_{i, 2}, \ldots, h_{i, N}\right]^{T}$, which denotes the transmission coefficients from $T_{i}$ to every antenna of the destination. The nodes in $\mathcal{R}$ re-encode the message and forward it to $D$, and each node $k$ in $\mathcal{J}$ attempts to confuse the eavesdroppers by injecting independent artificial noise $z_{k}$. We define the jammer and relay transmission matrices as $\mathbf{H}_{J}=\mathbf{H} \boldsymbol{\Lambda}$ and $\mathbf{H}_{R}=\mathbf{H}(\mathbf{I}-\boldsymbol{\Lambda})$, respectively, where $\boldsymbol{\Lambda}=\operatorname{diag}(\mathbf{b})$, and $\mathbf{b}=\left[b_{1}, b_{2}, \ldots, b_{M}\right]$. When $T_{i}$ is selected as a jammer, $b_{i}=1$, otherwise, $b_{i}=0$. The number of relays and jammers are respectively defined as $|\mathcal{R}|=L_{1} \geq 1$ and $|\mathcal{J}|=L_{2}$, where $L_{2}=M-L_{1}$.

Both the relays and the jammers transmit with a power of $P$. Since there are $N$ antennas at the destination and the CSI from all the intermediate nodes is available, beamforming can be performed at $D$. Assuming a beamformer $\mathbf{w} \in \mathbb{C}^{\mathrm{N} \times 1}$ is used at $D$, the signal at the destination is expressed by

$$
y_{D}=\sqrt{P} \mathbf{w}^{H} \mathbf{H}_{R} \mathbf{s}+\sqrt{P} \mathbf{w}^{H} \mathbf{H}_{J} \mathbf{z}+\mathbf{w}^{H} \mathbf{n}_{D},
$$

where $\mathbf{s}=[s, s, \ldots, s]^{T}, \mathbf{z}=\left[z_{1}, z_{2}, \ldots, z_{M}\right]^{T}, \mathbf{n}_{D}=$ $\left[n_{D_{1}}, n_{D_{2}}, \ldots, n_{D_{N}}\right]^{T}$, and $n_{D_{i}}$ is the noise at the $i$ th antenna of $D$. If $T_{i}$ is chosen as a relay, then $z_{i}=0$; otherwise, the non-zero entries of $\mathbf{z}$ are assumed to have unit power. The received $\mathrm{SNR}$ at the destination is

$$
\gamma_{D}=\frac{P \mathbf{w}^{H} \mathbf{H}_{R} \mathbf{e e}^{H} \mathbf{H}_{R}^{H} \mathbf{w}}{P \mathbf{w}^{H} \mathbf{H}_{J} \mathbf{H}_{J}^{H} \mathbf{w}+N_{0} \mathbf{w}^{H} \mathbf{w}},
$$

where $\mathbf{e}=[1,1, \ldots, 1]^{T} \in \mathbb{C}^{M \times 1}$. The achievable rate of the legitimate link is given by

$$
R_{D}=\frac{1}{2} \log _{2}\left(1+\gamma_{D}\right) .
$$

In the next section, we will present methods that prevent the eavesdroppers from identifying whether an intermediate node is a jammer or a relay. Thus, the eavesdroppers cannot perform coordinated beamforming, and must each attempt to individually intercept the transmitted signal. The SNR at $E_{j}$ is no more than

$$
\gamma_{E_{j}}=\frac{P\left(\left|g_{1, j}+g_{2, j}+\cdots+g_{L_{1}, j}\right|^{2}\right)}{P\left(\left|g_{L_{1}+1, j}\right|^{2}+\cdots+\left|g_{M, j}\right|^{2}\right)+N_{0}} .
$$

Define the maximum SNR among the eavesdroppers as $\gamma_{E}=$ $\max \gamma_{E_{j}}$, so the worst-case achievable rate at any of the eavesdroppers is

$$
R_{E}=\frac{1}{2} \log _{2}\left(1+\gamma_{E}\right) .
$$

Thus the achievable secrecy rate is no less than

$$
R_{s}=\left[R_{D}-R_{E}\right]^{+}=\left[\frac{1}{2} \log _{2}\left(\frac{1+\gamma_{D}}{1+\gamma_{E}}\right)\right]^{+},
$$

where $[z]^{+}=\max (0, z)$, and the SOP is given by

$$
P_{\text {out }}=\operatorname{Pr}\left(R_{s}<R\right)
$$

where $R$ is the required rate. Therefore, the relay and jammer selection problem for minimizing SOP can be written as

$$
\min _{\mathbf{b}} P_{\text {out }} \text {. }
$$

\section{SECURE RELAY AND JAMMER SELECTION}

In this section, we develop methods for relay and jammer selection that remain hidden from the eavesdroppers. The selection process consists of two steps. First, the destination determines appropriate relay and jammer sets using instantaneous CSI of the intermediate nodes and statistical CSI of the eavesdroppers. Second, the destination informs the intermediate nodes of the selection without giving away the information to the eavesdroppers. In the methods we present below, the destination broadcasts a vector and a corresponding selection threshold $T_{h r}$. Each node uses the vector to compute a channel-dependent quantity that is compared against the threshold; if it is above the threshold, then the node acts as a relay, otherwise it acts as a jammer. Note that knowledge of the vector and $T_{h r}$ at the eavesdroppers does not provide them any knowledge of the node selection, since they are not aware of the channels $\mathbf{h}_{j}$.

\section{A. Secrecy Outage Probability}

The SOP for our scenario is quantified below.

Proposition 1: The c.d.f. of $\gamma_{E_{j}}$ can be expressed by:

$$
\begin{aligned}
F_{\gamma_{E_{j}}}(z)= & 1-\frac{e^{\eta / \lambda_{g_{j}}}}{\lambda_{g_{j}}^{L_{2}}\left(L_{2}-1\right) !} \sum_{m=0}^{L_{2}-1}\left(\begin{array}{c}
L_{2}-1 \\
m
\end{array}\right)(-\eta)^{L_{2}-1-m} \\
& \times\left(\frac{L_{1} \lambda_{g_{j}}}{z+L_{1}}\right)^{(m+1)} \Gamma\left(m+1, \frac{\eta\left(z+L_{1}\right)}{L_{1} \lambda_{g_{j}}}\right), z \geq 0
\end{aligned}
$$


for $L_{2}>0$ where $\eta=N_{0} / P$, and $\Gamma(s, x)=\int_{x}^{\infty} t^{s-1} e^{-t} d t$ is the Upper Incomplete Gamma Function. For $L_{2}=0$ the c.d.f is given by

$$
F_{\gamma_{E_{j}}}(z)=1-e^{-\frac{z \eta}{M \lambda g_{j}}}, z \geq 0
$$

Proof: See Appendix A.

Note that $Z_{1}, Z_{2}, \ldots, Z_{K}$ are independent, so the c.d.f. of $\gamma_{E}$ can be obtained as $F_{\gamma_{E}}(z)=\prod_{j=1}^{K} F_{\gamma_{E_{j}}}(z)$, and the SOP of the system can be expressed by

$$
\begin{aligned}
P_{\text {out }} & =\operatorname{Pr}\left(R_{s}<R\right) \\
& =\operatorname{Pr}\left\{\frac{1}{2} \log _{2}\left(\frac{1+\gamma_{D}}{1+\gamma_{E}}\right)<R\right\} \\
& =1-\operatorname{Pr}\left\{\gamma_{E} \leq \frac{1+\gamma_{D}}{2^{2 R}}-1\right\} \\
& =1-F_{\gamma_{E}}\left(\frac{1+\gamma_{D}}{2^{2 R}}-1\right) .
\end{aligned}
$$

Given (11), once the relays and jammers are determined, the exact SOP can be calculated. Note that the SOP in (11) is monotonically decreasing with increases in $\gamma_{D}$ if $L_{1}$ (or equivalently $L_{2}$ ) is fixed. On the other hand, $P_{\text {out }}$ will increase as $K$ grows, approaching unity as $K \rightarrow \infty$.

\section{B. Selection of Relay and Jammer Sets}

It can be observed from (11) that $P_{\text {out }}$ is a function of $\gamma_{D}$ and $L_{2}$ when $M$ and $\lambda_{g_{j}}$ are known. Due to this fact, a joint optimization over $\gamma_{D}$ and $L_{2}$ can minimize the SOP. Considering $L_{2}$ is a finite integer, in this work we separate this joint problem into two steps. The first step is to minimize $P_{\text {out }}$ for any given $L_{2}$, which is equivalent to maximizing $\gamma_{D}$ for fixed $L_{2}$. Then the second step is to find the optimal $L_{2}$ that achieves the minimum $P_{\text {out }}$.

According to (2), maximizing $\gamma_{D}$ is equivalent to

$$
\max \frac{P \mathbf{w}^{H} \mathbf{H} \overline{\mathbf{b}} \overline{\mathbf{b}}^{H} \mathbf{H}^{H} \mathbf{w}}{\mathbf{w}^{H}\left(P \mathbf{H} \mathbf{\Lambda} \mathbf{H}^{H}+N_{0} \mathbf{I}\right) \mathbf{w}},
$$

where $\overline{\mathbf{b}}$ is defined as the logical complement of $\mathbf{b}$, i.e., $\overline{\mathbf{b}}:=$ $1-\mathbf{b}$. For a particular $\boldsymbol{\Lambda},(12)$ is a typical Rayleigh quotient problem, and the maximum value of $\gamma_{D}$ is

$$
\gamma_{D}{ }^{*}=\zeta_{\max }(\mathbf{Q})
$$

where $\mathbf{Q}=\left(P \mathbf{H} \mathbf{\Lambda} \mathbf{H}^{H}+N_{0} \mathbf{I}\right)^{-1}\left(P \mathbf{H} \overline{\mathbf{b}} \overline{\mathbf{b}}^{H} \mathbf{H}^{H}\right), \zeta_{\max }(\mathbf{Q})$ denotes the largest eigenvalue of matrix $\mathbf{Q}$, and $\mathbf{w}^{*}$ is given by

$$
\mathbf{w}^{*}=u_{\max }(\mathbf{Q}),
$$

where $u_{\max }(\mathbf{Q})$ denotes the eigenvector corresponding to $\zeta_{\max }(\mathbf{Q})$. Substituting $\gamma_{D}{ }^{*}$ into (11), the SOP corresponding to $\boldsymbol{\Lambda}$ can be calculated. Thus the SOP minimization problem of (8) can in principle be solved by an exhaustive search, although with a high computational complexity.

Selecting more nodes as relays may give a larger value of $\gamma_{D}$, but it also increases the risk that an eavesdropper succeeds in intercepting the source message through the wiretap link. Thus whether a node should be selected as a relay depends on its impact on both the destination and the eavesdroppers.
Assuming that we are in the more interesting situation where the jamming power dominates the background noise, then according to (12), maximizing $\gamma_{D}$ can be simplified to

$$
\begin{gathered}
\max _{\mathbf{w}, \boldsymbol{\Lambda}} \frac{\mathbf{w}^{H} \mathbf{H} \overline{\mathbf{b}} \overline{\mathbf{b}}^{H} \mathbf{H}^{H} \mathbf{w}}{\mathbf{w}^{H} \mathbf{H} \mathbf{\Lambda} \mathbf{H}^{H} \mathbf{w}} \\
\text { s.t. } \quad L_{2}=c_{0},
\end{gathered}
$$

where $c_{0} \in\{1, \ldots, M-1\}$ is fixed for the moment. With a given $\mathbf{w}$, it can be obtained from (15) that

$$
\begin{aligned}
\max \gamma_{D}= & \max \frac{\operatorname{trace}\left(\overline{\mathbf{b}} \overline{\mathbf{b}}^{H} \mathbf{H}^{H} \mathbf{w} \mathbf{w}^{H} \mathbf{H}\right)}{\operatorname{trace}\left(\mathbf{\Lambda} \mathbf{H}^{H} \mathbf{w} \mathbf{w}^{H} \mathbf{H}\right)} \\
= & \max \frac{\sum_{i=1}^{M} \bar{b}_{i}\left|\mathbf{w}^{H} \mathbf{h}_{i}\right|^{2}}{\sum_{i=1}^{M} b_{i}\left|\mathbf{w}^{H} \mathbf{h}_{i}\right|^{2}} \\
& +\frac{\sum_{i, j=1, i \neq j}^{M} \bar{b}_{i} \bar{b}_{j} \operatorname{Re}\left\{\left(\mathbf{w}^{H} \mathbf{h}_{i}\right)^{H}\left(\mathbf{w}^{H} \mathbf{h}_{j}\right)\right\}}{\sum_{i=1}^{M} b_{i}\left|\mathbf{w}^{H} \mathbf{h}_{i}\right|^{2}} \\
& \geq \max \frac{\sum_{i=1}^{M} \bar{b}_{i}\left|\mathbf{w}^{H} \mathbf{h}_{i}\right|^{2}}{\sum_{i=1}^{M} b_{i}\left|\mathbf{w}^{H} \mathbf{h}_{i}\right|^{2}} .
\end{aligned}
$$

Eq. (16) gives a lower bound for the maximum value of $\gamma_{D}$, which suggests that the intermediate nodes with the $L_{2}$ minimum values of $\left|\mathbf{w}^{H} \mathbf{h}_{i}\right|$ should be selected as jammers, and the rest selected as relays. Without loss of generality, reorder $\left|\mathbf{w}^{H} \mathbf{h}_{i}\right|$ in increasing order, set $T_{h r}$ as any value between $\left|\mathbf{w}^{H} \mathbf{h}_{L_{2}}\right|$ and $\left|\mathbf{w}^{H} \mathbf{h}_{L_{2}+1}\right|$, and then the jammer set can be obtained as $\mathcal{J}=\left\{T_{i}:\left|\mathbf{w}^{H} \mathbf{h}_{i}\right|<T_{h r}\right\}$. To define the selection result $\boldsymbol{\Lambda}$, assign $b_{i}=1$ when $T_{i}$ is a jammer and $b_{i}=0$ otherwise.

To solve the complete problem, the destination must not only find $\mathbf{w}$, but also the optimal value for $L_{2}$ that minimizes the SOP. Once this is done, it will feed back the vector $\mathbf{w}$ and the corresponding threshold $T_{h r}$ to each intermediate node. Using its CSI to the destination, each node $i$ then compares $\left|\mathbf{w}^{H} \mathbf{h}_{i}\right|$ to the threshold to determine its action. While an exhaustive search could be used to find globally optimal values for $\mathbf{w}$ and $L_{2}$, we propose two simpler solutions below that perform nearly as well at a fraction of the computational cost.

1) Greedy Method: In the Greedy Method (GM), we begin by assuming no jammers, i.e., $\mathbf{b}=\mathbf{0}$, in which case $\mathbf{Q}=$ $\mathbf{H e e}^{H} \mathbf{H}^{H}$ and $\mathbf{w}=u_{\max }(\mathbf{Q})$. We then set $L_{2}=1$, and find the node with the smallest value for $\left|\mathbf{w}^{H} \mathbf{h}_{i}\right|$. This node is placed in the jammer set, a new $\mathbf{w}$ is calculated, and the corresponding SOP is determined. Next we set $L_{2}=2$ and find the node in the relay set for which $\left|\mathbf{w}^{H} \mathbf{h}_{i}\right|$ is smallest, switch it to the jamming set and update $\mathbf{w}$ and the SOP. This process is repeated until only one relay is left, and the value for $L_{2}$ is chosen to be the one that results in the smallest SOP. Compared with the exhaustive search, which requires checking all $2^{M}-1$ possible selection results, the GM only requires $M$ SOP evaluations.

2) Vector Alignment Method: The basic idea of the Vector Alignment Method (VAM) is to group intermediate nodes together whose channel vectors are most aligned. For example, if $T_{i}$ is selected as a relay since $\left|\mathbf{w}^{H} \mathbf{h}_{i}\right|$ is relatively large, then nodes with channel vectors $\mathbf{h}_{j}$ that are "aligned" or nearly parallel with $\mathbf{h}_{i}$ should also be chosen as relays. This method is implemented as follows. For every node $T_{i}$ and every possible value of $L_{1}$, we calculate the SOP assuming $T_{i} \in \mathcal{R}$ and the 


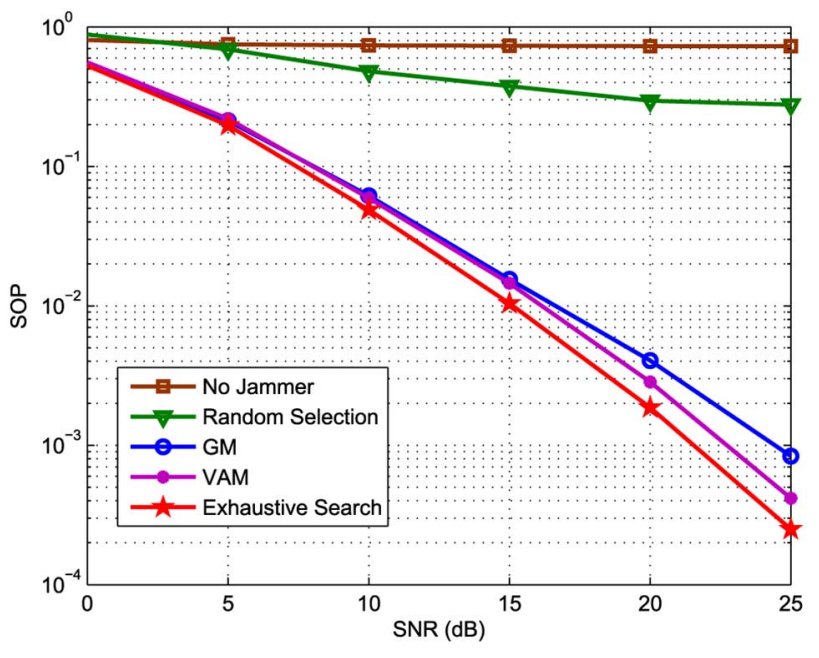

Fig. 1. Secrecy outage probability for different selection methods.

other relays are chosen as the $L_{1}-1$ nodes whose channel vectors $\mathbf{h}_{j}$ maximize $c_{i j}=\left|\mathbf{h}_{i}^{H} \mathbf{h}_{j}\right| /\left(\left|\mathbf{h}_{i}\right|\left|\mathbf{h}_{j}\right|\right)$, the cosine of the angle between the vectors. The VAM method is somewhat more costly than GM, requiring that the SOP be calculated for $M(M$ $-1)+1$ possible scenarios (including the case where $\mathbf{b}=0$ ), but still significantly less costly than an exhaustive search when $M$ is large.

\section{Simulation Results}

In this section, simulation results are presented to illustrate the SOP of the proposed methods. We set $\lambda_{h_{i, j}}=1, \lambda_{g_{i, j}}=1$, and $R=1$ for all simulations. In Fig. 1, the SOP is plotted for a case where $M=4, N=3$, and $K=2$. It is observed that the SOP achieved with all nodes acting as relays is close to 1 , which shows that without jamming the "best" eavesdropper can on average achieve nearly the same SNR as the destination. While using a random node assignment provides an improvement over using no cooperative jamming, the GM and VAM methods perform significantly better, and their SOP performance is nearly as good as that achieved by an exhaustive search.

Fig. 2 shows the performance of the GM and VAM methods compared with "insecure" relay and jammer selection $(M=3$, $N=3$ ), where the eavesdroppers are aware of the relay/jammer status of the intermediate nodes and can cooperatively aggregate the signals of interest and cancel the jamming noise in a manner similar to the destination. We see that both GM and VAM have significantly lower SOP, even when they encounter twice as many eavesdroppers as in the case of an insecure selection.

\section{CONCLUSIONS}

This paper has studied secure relay and jammer selection for minimizing the secrecy outage probability in cooperative wireless networks. A closed-form expression for the SOP was derived, and methods were developed that allow for cooperative nodes to be assigned as either relays or jammers in an attempt to minimize the resulting SOP. Most importantly, the algorithms are implemented in such a way that the node assignment can not be determined by the eavesdroppers, preventing

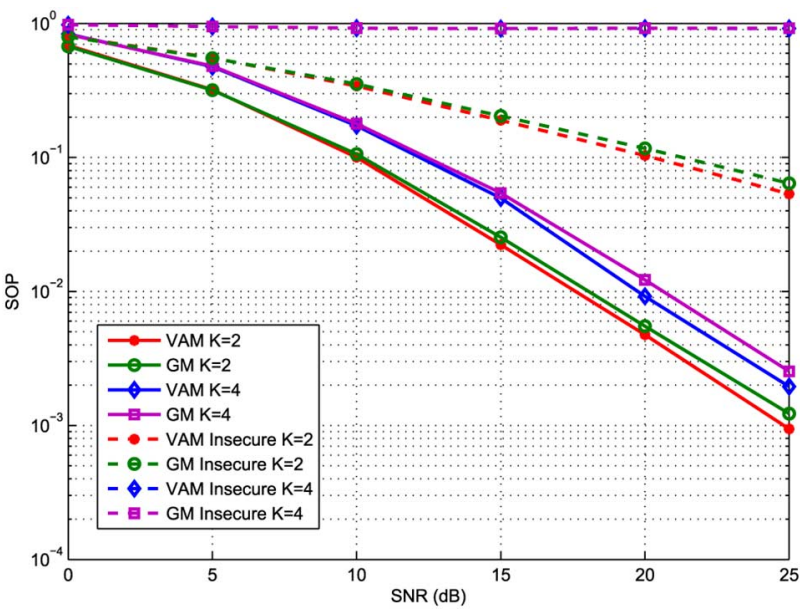

Fig. 2. Secrecy outage probability for secure and insecure selections.

them from cooperatively using distributed multi-antenna techniques to null the interference. Besides an optimal method based on an exhaustive search for the best node assignment, reduced complexity methods were discussed that have SOP performance approaching that of the exhaustive search. Simulations results were presented to show the advantage of the proposed methods compared with a random assignment or with an assignment that was not kept secret from the eavesdroppers.

APPENDIX A

ProOF OF PROPOSITION 1

For $L_{2}>0$, let $X=\left|\sum_{i=1}^{L_{1}} g_{i, j}\right|^{2}$ and $U$ $=\sum_{i=L_{1}+1}^{M}\left|g_{i, j}\right|^{2}$. It can be found that $X \sim \exp \left(1 /\left(L_{1} \lambda_{g_{j}}\right)\right)$ and that $U$ follows a central chi-square distribution with $2 L_{2}$ degrees of freedom. Denoting $\gamma_{E_{j}}=\frac{X}{U+N_{0} / P}$ and $Y=U$ $+\eta$, we have

$$
F_{\gamma_{E_{j}}}(z)=\int_{\eta}^{\infty} f_{Y}(y) F_{X}(y z) d y .
$$

According to the c.d.f. of $X$, we have

$$
F_{X}(y z)=1-e^{-\frac{y z}{L_{1} \lambda g_{j}}},
$$

and using the p.d.f. of $U$, we obtain

$$
f_{Y}(y)=\frac{d F_{Y}(y)}{d y}=f_{U}(y-\eta)=\frac{(y-\eta)^{L_{2}-1}}{\lambda_{g_{j}}^{L_{2}}\left(L_{2}-1\right) !} e^{-(y-\eta) / \lambda_{g_{j}}}
$$

Substituting (18) and (19) into (17), it can be seen that

$$
\begin{aligned}
F_{\gamma_{E_{j}}}(z)= & 1-\frac{e^{\eta / \lambda_{g_{j}}}}{\lambda_{g_{j}}^{L_{2}}\left(L_{2}-1\right) !} \\
& \times \int_{\eta}^{\infty}(y-\eta)^{L_{2}-1} e^{-\left(z+L_{1}\right) y / L_{1} / \lambda_{g_{j}}} d y .
\end{aligned}
$$

Applying the Binomial Theorem and the Upper Incomplete Gamma Function, we finally obtain (9). For $L_{2}=0,(10)$ can be derived similarly. 


\section{REFERENCES}

[1] H. Delfs and H. Knebl, Introduction to Cryptography: Principles and Applications, 2nd ed. Berlin, Germany: Springer, 2007.

[2] A. D. Wyner, "The wire-tap channel," Bell Syst. Techn. J., vol. 54, no. 8, pp. 1355-1387, 1975.

[3] S. K. Leung-Yan-Cheong and M. E. Hellman, "The Gaussian wire-tap channel," IEEE Trans. Inf. Theory, vol. 24, no. 4, pp. 451-456, 1978.

[4] L. Dong, Z. Han, A. P. Petropulu, and H. V. Poor, "Improving wireless physical layer security via cooperating relays," IEEE Trans. Signal Process., vol. 58, no. 3, pp. 1875-1888, 2010.

[5] E. Tekin and A. Yener, "The general gaussian multiple-access and two-way wiretap channels: Achievable rates and cooperative jamming," IEEE Trans. Inf. Theory, vol. 54, no. 6, pp. 2735-2751, 2008.

[6] J. Wang and A. Swindlehurst, "Cooperative jamming in MIMO ad-hoc networks," in Proc. Asilomar Conf. on Signals, Systems and Computers, Nov. 2009, pp. 1719-1723.

[7] S. A. A. Fakoorian and A. Swindlehurst, "Solutions for the MIMO gaussian wiretap channel with a cooperative jammer," IEEE Trans. Signal Process., vol. 59, no. 10, pp. 5013-5022, Oct. 2011.

[8] J. Huang and A. L. Swindlehurst, "Cooperative jamming for secure communications in MIMO relay networks," IEEE Trans. Signal Process., vol. 59, no. 10, pp. 4871-4884, 2011.

[9] Y. Liu, J. Li, and A. P. Petropulu, "Destination assisted cooperative jamming for wireless physical-layer security," IEEE Trans. Inf. Forensics Secur., vol. 8, no. 4, pp. 682-694, 2013.
[10] J. Li, A. P. Petropulu, and S. Weber, "On cooperative relaying schemes for wireless physical layer security," IEEE Trans. Signal Process., vol. 59, no. 10, pp. 4985-4997, 2011.

[11] Y. Zou, X. Wang, and W. Shen, "Optimal relay selection for physicallayer security in cooperative wireless networks," IEEE J. Sel. Areas Commun., vol. 31, no. 10, pp. 2099-2111, 2013.

[12] I. Krikidis, J. S. Thompson, and S. McLaughlin, "Relay selection for secure cooperative networks with jamming," IEEE Trans. Wireless Commun., vol. 8, no. 10, pp. 5003-5011, 2009.

[13] V. Bao, N. Linh-Trung, and M. Debbah, "Relay selection schemes for dual-hop networks under security constraints with multiple eavesdroppers," IEEE Trans. Wireless Commun., vol. 12, no. 12, pp. 6076-6085, 2013.

[14] J. Chen, L. Song, and Z. Han et al., "Joint relay and jammer selection for secure decode-and-forward two-way relay communications," in IEEE Global Telecommunications Conf. (Globecom), Houston, USA, Dec. 2011, pp. 1-5.

[15] J. Chen, R. Zhang, and L. Song et al., "Joint relay and jammer selection for secure two-way relay networks," IEEE Trans. Inf. Forensics Secur., vol. 7, no. 1, pp. 310-320, 2012.

[16] M. Lin, J. Ge, and Y. Yang et al., "Joint cooperative beamforming and artificial noise design for secrecy sum rate maximization in two-way AF relay networks," IEEE Commun. Lett., vol. 18, no. 2, pp. 380-383, 2014.

[17] Z. Ding, M. Xu, and J. Lu et al., "Improving wireless security for bidirectional communication scenarios," IEEE Trans. Veh. Technol., vol. 61, no. 6, pp. 2842-2848, 2012. 